\title{
Chemotherapy-induced nausea and vomiting in daily clinical practice: a community hospital-based study
}

\author{
Doranne L. Hilarius • Paul H. Kloeg • \\ Elsken van der Wall • Joris J. G. van den Heuvel • \\ Chad M. Gundy $\cdot$ Neil K. Aaronson
}

Received: 15 September 2010 / Accepted: 21 December 2010 / Published online: 22 January 2011

(C) The Author(s) 2011. This article is published with open access at Springerlink.com

\begin{abstract}
Background Chemotherapy-induced nausea and vomiting (CINV) are major adverse effects of cancer chemotherapy. This study investigated: (1) the impact of CINV on patients' health-related quality of life (HRQL) in daily clinical practice; (2) the association between patient characteristics and type of antiemetics and CINV; and (3) the role of CINV in physicians' decisions to modify antiemetic treatment.

Patients and methods This prospective, multicenter study was conducted in nine general hospitals in the Netherlands. During three consecutive chemotherapy cycles, patients used a diary to record episodes of nausea, vomiting and
\end{abstract}

\section{L. Hilarius}

Red Cross Hospital,

Vondellaan 13,

1942 LE Beverwijk, The Netherlands

P. H. Kloeg

Medical Center Alkmaar,

Wilhelminalaan 12 ,

1815 JD Alkmaar, The Netherlands

E. van der Wall

Division of Internal Medicine and Dermatology,

University Medical Center Utrecht,

G02.228, PO Box 85500, 3508 GA Utrecht, The Netherlands

J. J. G. van den Heuvel

Sint Lucas Andreas Hospital,

Jan Tooropstraat 164,

1061 AE Amsterdam, The Netherlands

C. M. Gundy • N. K. Aaronson $(\bowtie)$

Division of Psychosocial Research and Epidemiology,

The Netherlands Cancer Institute,

Plesmanlaan121,

1066 CK Amsterdam, The Netherlands

e-mail: n.aaronson@nki.nl antiemetic use. For each cycle, these ratings were made 1 day prior to and 7 days after having received chemotherapy. The influence of CINV on patients' HRQL was evaluated with the Functional Living Index-Emesis (FLIE) questionnaire at day 6 of each treatment cycle. (Changes in) antiemetic use were recorded by the treating nurse. Patient inclusion took place between May 2005 and May 2007. Results Two hundred seventy-seven patients were enrolled in the study. Acute and delayed nausea during the first treatment cycle was reported by $39 \%$ and $68 \%$ of the patients, respectively. The comparable figures for acute and delayed vomiting were $12 \%$ and $23 \%$. During the first and subsequent treatment cycle, approximately one-third of the patients indicated that CINV had a substantial impact on their daily lives. Female patients and younger patients reported significantly more CINV than male and older patients. At all treatment cycles, patients receiving treatment with moderately emetogenic chemotherapy, containing anthracycline, reported more acute nausea than patients receiving highly emetogenic chemotherapy. Acute vomiting was associated significantly with change in (i.e., additional) antiemetic treatment. Delayed CINV did not influence antiemetic treatment.

Conclusion CINV continues to be a problem that adversely affects the daily lives of patients. CINV is worse in women and in younger patients. In daily clinical practice, acute CINV, but not delayed CINV, results in changes in antiemetic treatment. In view of the effects of not only acute, but also delayed CINV on daily life, more attention should be paid to adjustment of antiemetic treatment to cover CINV complaints, later during the chemotherapy cycle.

Keywords Chemotherapy-induced nausea and vomiting . Antiemetics · Symptom management 


\section{Introduction}

Nausea and vomiting are the most frequently reported adverse effects of antineoplastic chemotherapy and significantly affect patients' daily functioning and health-related quality of life (HRQL) [1]. The development of the 5HT3 antagonists represents a significant advance in preventing chemotherapy-induced nausea and vomiting (CINV) [2]. More recently, aprepitant, which selectively blocks the binding of substance P at the NK-1 receptor in the central nervous system, has been shown to augment the antiemetic activity of the 5HT3 antagonists and of the corticosteroid dexamethasone, and thus, to inhibit both acute (i.e., during or immediately following a chemotherapy cycle) and delayed (i.e., after chemotherapy) emesis of highly emetogenic chemotherapy $[3,4]$.

Most studies of the effects of CINV on daily life are characterized by narrow patient selection criteria and are limited to well-defined chemotherapy and antiemetic regimens. In addition, most of these studies have been limited to only a single treatment cycle and were performed in specialized cancer centers, rather than community hospitals where the majority of cancer patients are treated.

A recent study, focusing on the effects of chemotherapy on CINV, and the effect of CINV on daily life found that $36 \%$ of all patients reported emesis and $60 \%$ reported nausea. Patients treated with highly emetogenic chemotherapy (HEC) reported significantly lower mean HRQL compared to patients treated with moderately emetogenic chemotherapy (MEC) [5]. However, this study was limited to 1 cycle of chemotherapy, and the association between patient characteristics and CINV was not reported.

Retrospective analyses performed on some of the earliest antiemetic trials, identified a number of patient and treatment characteristics that appear to have predictive value for the development of CINV [6-9]. Subsequent trials evaluating 5HT3 antagonists have, for the most part, confirmed the predictive value of these factors, including age, sex, alcohol use, history of motion sickness, and dose of cisplatin [10-12]. Although many of these factors are mentioned in international guidelines on the treatment of CINV, little is known about their impact on CINV in daily clinical practice among unselected patients receiving different types of chemotherapy and antiemetics [1].

Grunberg et al. found that physicians and nurses could accurately report the incidence of acute CINV but often (in $75 \%$ of the cases) underestimated the incidence of delayed nausea and emesis after chemotherapy [13]. This would suggest that delayed CINV is not well managed, as awareness of the problem is a sine qua non for effective treatment. In randomized clinical trials on the effectiveness of antiemetics, the use of the antiemetic schedules is predefined. Adjustment of antiemetic treatment, based on the actual level of CINV experienced by the patients, has not typically been part of the study protocols. So, in fact, little is known about the adequacy of treatment of CINV throughout the course of chemotherapy treatment. More specifically, little is known about the adjustments that are made in the choice of antiemetics in daily clinical practice based on patient's self-reported symptoms.

Against this background, we designed our study to: (1) determine the incidence of CINV during consecutive treatment cycles in daily clinical practice in general hospitals; (2) investigate the association between patient characteristics and types of antiemetics, CINV, and HRQL; and (3) examine the influence of CINV on changes in antiemetic therapy, and specifically on adding antiemetics, over multiple cycles of chemotherapy.

\section{Methods}

Study design and setting

This prospective, multicenter, observational study was conducted in the departments of medical oncology of nine hospitals in the wider Amsterdam region of the Netherlands (see Appendix).

\section{Patient sample}

The study sample was composed of a consecutive series of chemotherapy naive patients treated during a 2-year period. Patients had to be scheduled for treatment with either HEC, MEC containing anthracycline, or MEC not containing anthracycline (see paragraph 'Data collection and study measures' for more details). Patients were excluded if they lacked basic proficiency in the Dutch language, if they were younger than 18 years of age, or exhibited overt psychopathology or serious cognitive problems that would impede their ability to take part in the study. Eligible patients were given both oral and written information about the study prior to obtaining their informed consent. The institutional review boards of the participating hospitals approved the study. Patient inclusion took place between May 2005 and May 2007.

Data collection and study measures

Patients recorded episodes of nausea, vomiting, and use of antiemetics in a diary for the day prior to and for 7 days following each of three consecutive chemotherapy cycles. Patients were instructed to use the diary everyday to record each emetic episode and to provide daily nausea assessments using a $100-\mathrm{mm}$ visual analogue scale (VAS) to rate 
the severity of nausea experienced during the preceding $24 \mathrm{~h}$. Patients also recorded all rescue antiemetic medication taken in addition to what was prescribed at baseline to prevent nausea and vomiting. Only the research team had access to the patient diaries. Treatment decisions were based on the physicians' usual patient assessment practice.

Using previously published decision rules, no nausea was defined as a VAS score of less than $5 \mathrm{~mm}$ on the $100-\mathrm{mm}$ scale. A patient was considered to have had acute nausea or acute emesis if nausea (VAS $>=5 \mathrm{~mm}$ ), or at least one episode of vomiting was reported during the first $24 \mathrm{~h}$ after start of chemotherapy [3, 5, 14]. Any episode of nausea and/or vomiting thereafter up to 5 days after chemotherapy was considered delayed $[5,14,15]$.

In addition, on day 5 after each chemotherapy cycle, patients were asked to complete the 18-item Functional Living Index-Emesis (FLIE), a validated self-report questionnaire designed to evaluate the impact of CINV on patients' daily lives [16, 17] Response categories range from one to seven on a modified linear analogue scale. The total score, calculated by summing the responses to the 18 items, ranges from 18 to 126 , with higher scores representing better health outcomes (i.e., less impact of CINV on daily life) [18]. Following Bloechl-Daum et al., an individual item score of six or more, and a total FLIE score of 108 or more were considered evidence of no or minimal impact of CINV on daily life [5].

Sociodemographic and clinical information were obtained via interview, nurse ratings, and/or from the medical records. This included age, sex, country of origin, WHO performance status, alcohol intake, prior pregnancy-related emesis, problems with motion sickness, cancer diagnosis, type of chemotherapy, radiotherapy, and emetogenicity.

Emetogenicity was defined as HEC (i.e., at least one chemotherapeutic agent of Hesketh level 5 emetic potential, such as cisplatin in combination with other chemotherapy), MEC containing anthracycline (i.e.. at least one anthracycline (doxorubicin, epirubicin) in combination with non- platinum containing chemotherapy), or MEC not containing anthracycline (i.e., at least one chemotherapeutic agent of Hesketh level 3 or 4 emetic potential) $[15,19]$ Prescribed antiemetics were recorded by the nurses at each chemotherapy cycle.

For interpretation of the use of antiemetics, combinations of antiemetics were categorized in accordance with international guidelines. Table 1 summarizes the 2007 NCCN guidelines on antiemetics. From these guidelines, six combinations can be defined:

a a combination of a 5HT3 antagonist, a corticosteroid and aprepitant

b a combination of a 5HT3 antagonist and a corticosteroid

c a 5 HT3 antagonist

d a corticosteroid

e aprepitant and a corticosteroid

$\mathrm{r}$ other antiemetics (for example metoclopramide or a benzodiazepine)

\section{Statistical analysis}

A non-respondent analysis using logistic regression was performed to compare patient and treatment characteristics of patients returning all three diaries with those of patients returning less than three diaries.

Descriptive statistics (means, standard deviations, percentages) are reported for patient background characteristics and prevalence of CINV. To evaluate the influence of patient characteristics and type of antiemetic on CINV, a series of logistic regression analyses was performed with acute nausea, delayed nausea, acute vomiting, delayed vomiting, and FLIE scores as dependent variables, and age, gender, emetogenicity, antiemetic schedule, and alcohol use as independent variables.

Logistic regression analysis was also used to evaluate the influence of patient and treatment characteristics on
Table 1 NCCN antiemesis guidelines[1]

\footnotetext{
${ }^{\text {a }}$ Aprepitant should be added to dexamethasone and a 5-HT3 antagonist for patients receiving the combination of an anthracycline and cyclophosphamide and selected patients receiving other chemotherapies of moderate emetic risk (e.g., carboplatin, doxorubicin, epirubicin, ifosfamide, irinotecan, methotrexate)
}

\begin{tabular}{|c|c|}
\hline Chemotherapy & Antiemetics \\
\hline \multicolumn{2}{|l|}{ Acute CINV (day 1) } \\
\hline High risk & $5 \mathrm{HT}_{3}$-antagonist + dexamethasone + aprepitant \\
\hline Moderate risk & $5 \mathrm{HT}_{3}$-antagonist + dexamethasone + aprepitant in selected patients ${ }^{\mathrm{a}}$ \\
\hline Low risk & dexamethasone or metoclopramide or prochlorperazine \\
\hline Minimal risk & No antiemetics \\
\hline \multicolumn{2}{|l|}{ Delayed CINV } \\
\hline High risk & $\begin{array}{l}\text { Dexamethasone (days 2-4)+aprepitant (days 2-3)+lorazepam in selected } \\
\text { patients }\end{array}$ \\
\hline Moderate risk & $\begin{array}{l}\text { Dexamethasone (days 2-4)+aprepitant if used on day } 1 \text { (days } 2-3)^{\mathrm{a}} \text { or } \\
5 \mathrm{HT}_{3} \text {-antagonist (days } 2-4 \text { ) +lorazepam in selected patients }\end{array}$ \\
\hline Low en minimal risk & No antiemetics \\
\hline
\end{tabular}


adding antiemetics across treatment cycles. Independent variables included age, gender, emetogenicity, CINV, and alcohol use. Where use of logistic regression was not possible due to missing cells, the chi-square statistic was used.

Differences in the prevalence of CINV and the impact of CINV on daily life over the three chemotherapy cycles were analyzed using McNemar's test for correlated proportions [20].

Results

\section{Patients}

Two hundred seventy-seven patients were enrolled in the study. During the course of the study, 52 patients were lost to follow-up. Five patients died, 4 found the study too burdensome, 15 did not complete or failed to return the diary, 6 became too ill to continue participation, 9 discontinued chemotherapy treatment, and 13 patients discontinued participation for unknown reasons (see Fig. 1). Data on at least one chemotherapy cycle were available for 273 patients, two cycles for 253 patients, and all three chemotherapy cycles for 225 patients.

Table 2 summarizes the sociodemographic and clinical characteristics of the study sample. The mean age was 56 years (range, 24-87 years). Approximately, one-third of

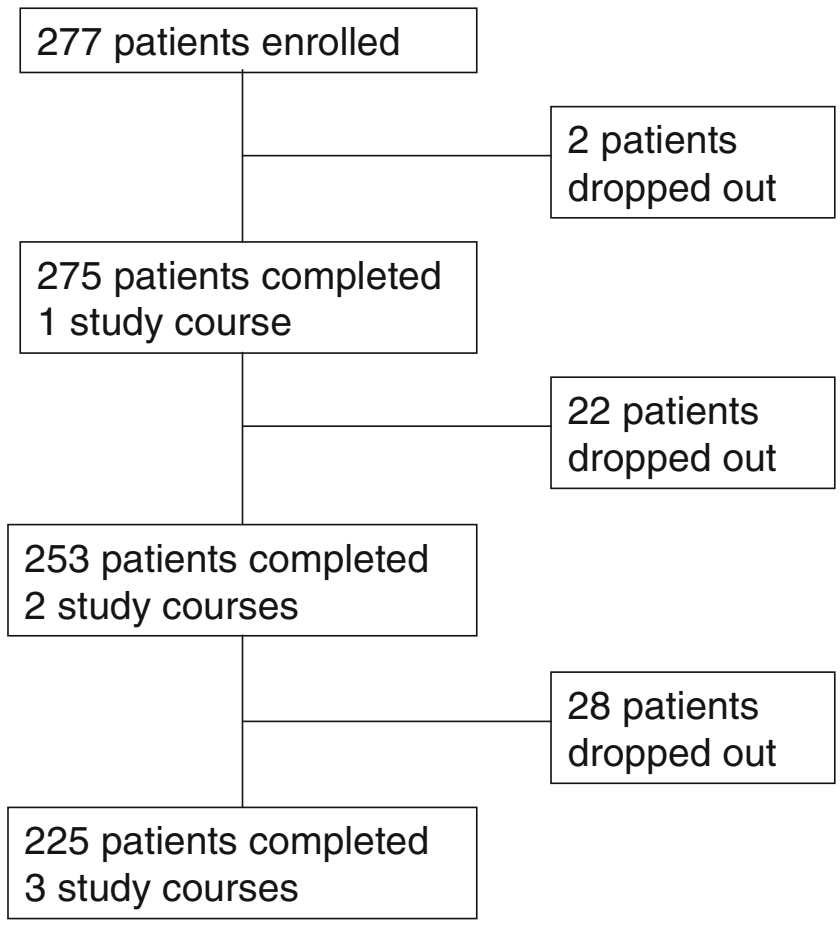

Fig. 1 Patient flow chart. Reasons for patient drop-out during the study: death $(n=5)$; study too burdensome $(n=4)$; too ill $(n=6)$; treatment stopped $(n=9)$; failure to return diary/unknown $(n=28)$ the patients were male. The most frequent diagnoses were breast $(46.2 \%)$, lung $(22.7 \%)$, and colorectal cancer (9.7\%). Thirty-seven percent of patients received HEC, $51 \%$ MEC, containing anthracyclines, and $12 \%$ MEC without anthracyclines.

Results of the non-respondent analysis indicated that in comparison to those patients returning all three diaries, those returning less than three diaries were significantly younger ( 55 vs 60 years, $p=0.02$ ), and were significantly more likely to have received highly emetogenic than moderately emetogenic chemotherapy $(84 \%$ vs $68 \%, p=$ 0.02 ). No significant differences were observed between full and partial respondents in gender, cancer diagnosis, WHO performance status, or alcohol use (data not shown in tabular form).

\section{Incidence of CINV and its effect on daily life (Table 3)}

Acute and delayed nausea during the first treatment cycle were reported by $39 \%$ and $68 \%$ of the patients, respectively. The comparable figures for acute and delayed vomiting were $12 \%$ and $23 \%$. Thirty-four percent of the patients indicated that, overall, CINV during the first treatment cycle had a significant impact on their daily life (i.e., a total FLIE score $\leq 108$ ). The percentage of patients suffering from CINV and/or reporting CINV, having an impact on daily life, did not change significantly at the second and third treatment cycle (Table 3).

\section{Age and CINV (Table 3)}

At all treatment cycles, significantly more younger patients $(<65)$ reported acute chemotherapy-induced nausea than older patients (first cycle $47 \%$ vs $14 \%, p<$ 0.005 ; second cycle $44 \%$ vs $21 \%, p<0.005$; third cycle 48 vs $20 \%, p<0.005)$. At all treatment cycles, younger patients experienced more delayed nausea than older patients; this was statistically significant for the second treatment cycle ( 71 vs $46 \%, p<0.005$ ). A similar pattern of results was observed for acute vomiting at the second and third treatment cycles, with significantly more younger patients reporting vomiting than older patients $(12 \%$ vs $3 \%, p=0.05$; and $11 \%$ vs $2 \%, p=0.05)$. At the second and the third treatment cycle, significantly more younger than older patients reported that CINV had an impact on their daily lives $(41 \%$ vs $21 \%, p=0.03$ and $44 \%$ vs $26 \%, p=0.05$ ).

\section{Gender and CINV (Table 3)}

At the first treatment cycle, female patients suffered significantly more from CINV than male patients (acute nausea $48 \%$ vs $18 \%, p<0.005$; delayed nausea $75 \%$ vs 
Table 2 Patient characteristics $(N=275)^{\mathrm{a}}$
No. of patients

Percentage (\%)

\begin{tabular}{|c|c|c|c|}
\hline & & No. of patients & Percentage (\%) \\
\hline \multirow[t]{2}{*}{ Sex } & Female & 190 & 69 \\
\hline & Male & 85 & 31 \\
\hline Age, mean (range) & & $56(24-87)$ & \\
\hline \multirow[t]{9}{*}{ Hospital } & 1 & 22 & 7.9 \\
\hline & 2 & 7 & 2.5 \\
\hline & 3 & 48 & 17.3 \\
\hline & 4 & 23 & 8.3 \\
\hline & 5 & 19 & 7.2 \\
\hline & 6 & 18 & 6.5 \\
\hline & 7 & 43 & 15.5 \\
\hline & 8 & 46 & 17.0 \\
\hline & 9 & 49 & 17.7 \\
\hline \multirow[t]{6}{*}{ WHO performance status } & 0 & 169 & 61.4 \\
\hline & 1 & 83 & 30.3 \\
\hline & 2 & 14 & 5.1 \\
\hline & 3 & 5 & 1.8 \\
\hline & 4 & 0 & 0 \\
\hline & unknown & 4 & 1.4 \\
\hline \multirow[t]{5}{*}{ Alcohol use } & 0 units/week & 108 & 39.4 \\
\hline & 1-4 units/week & 96 & 34.7 \\
\hline & 5-10 units/week & 34 & 12.6 \\
\hline & 11 or more units/week & 29 & 10.5 \\
\hline & Unknown & 8 & 2.9 \\
\hline \multirow[t]{7}{*}{ Cancer diagnosis } & Breast & 127 & 46.2 \\
\hline & Lung & 62 & 22.7 \\
\hline & Colorectal & 27 & 9.7 \\
\hline & Urogenital & 7 & 2.5 \\
\hline & Gynecological & 11 & 4.0 \\
\hline & Lymphoma & 17 & 6.1 \\
\hline & Other & 24 & 8.8 \\
\hline \multirow[t]{5}{*}{ Chemotherapy intent } & Curative & 59 & 21.7 \\
\hline & Adjuvant & 115 & 41.5 \\
\hline & Palliative & 76 & 27.8 \\
\hline & Neo-adjuvant & 18 & 6.5 \\
\hline & Unknown & 7 & 2.5 \\
\hline \multirow[t]{3}{*}{ Emetogenicity of chemotherapy } & High & 101 & 37 \\
\hline & Moderate (anthracycline) & 140 & 51 \\
\hline & Moderate (no anthracycline) & 34 & 12 \\
\hline History of pregnancy emesis & & 43 & 15.6 \\
\hline History of motion sickness & & 41 & 14.9 \\
\hline
\end{tabular}

${ }^{\mathrm{a}}$ The two patients that dropped out before completing one study course were not included in the analyses

\section{Alcohol use and CINV (Table 3)}

$51 \%, p=0.009$; acute vomiting $15 \%$ vs $5 \%, p=0,01$; delayed vomiting $27 \%$ vs $14 \%, p=0.05$ ). A similar pattern of results was observed at the second and the third treatment cycles. At the first treatment cycle, significantly more women than men reported that CINV had a negative impact on their daily lives ( $42 \%$ versus $22 \%, p=0.03$ ). Again, a similar pattern of results was observed at treatment cycles 2 and 3.
The association between alcohol use and CINV was not entirely consistent. At the first treatment cycle, patients who consumed more alcohol per week reported significantly less acute vomiting than patients consuming less $(p=0.03)$. This was also found for acute nausea at the third treatment cycle $(p=0.02)$. However, no other significant 
Table 3 Association between patient-related variables, CINV, and CINV effects on daily life

\begin{tabular}{|c|c|c|c|c|c|c|c|c|c|c|c|}
\hline & & \multicolumn{2}{|c|}{ Acute nausea } & \multicolumn{2}{|c|}{ Delayed nausea } & \multicolumn{2}{|c|}{ Acute vomiting } & \multicolumn{2}{|c|}{ Delayed vomiting } & \multicolumn{2}{|l|}{ FLIE $^{\mathrm{c}}$} \\
\hline & & $\mathrm{n}(\%)$ & $p$-value ${ }^{\mathrm{a}}$ & n $(\%)$ & $p$-value ${ }^{\mathrm{a}}$ & $\mathrm{n}(\%)$ & $p$-value ${ }^{\mathrm{b}}$ & $\mathrm{n}(\%)$ & $p$-value ${ }^{\mathrm{a}}$ & n $(\%)$ & $p$-value ${ }^{\mathrm{a}}$ \\
\hline \multicolumn{12}{|l|}{ First course $n=275$} \\
\hline \multirow[t]{2}{*}{ Gender } & male & $15(18)$ & & $44(51)$ & & $4(5)$ & & $12(14)$ & & $17(22)$ & \\
\hline & female & $92(48)$ & $<0,005$ & $144(75)$ & 0,009 & $29(15)$ & 0,01 & $51(27)$ & 0,05 & $78(42)$ & 0,03 \\
\hline \multirow[t]{3}{*}{ Emetogenicity } & HEC & $14(14)$ & & $57(56)$ & & $3(3)$ & & $19(19)$ & & $24(25)$ & \\
\hline & MEC (anthracycline) & $82(59)$ & & $105(75)$ & & $27(19)$ & & $37(26)$ & & $58(43)$ & \\
\hline & MEC (not anthracycline) & $11(31)$ & $<0,005$ & $26(72)$ & n.s. & $3(8)$ & $<0,005$ & $7(19)$ & n.s. & $13(45)$ & 0,02 \\
\hline \multirow[t]{2}{*}{ Age } & $<65$ & $96(47)$ & & $152(74)$ & & $27(13)$ & & $51(25)$ & & $78(40)$ & \\
\hline & $>65$ & $10(14)$ & $<0,005$ & $36(51)$ & n.s. & $5(7)$ & n.s. & $12(17)$ & n.s. & $16(24)$ & n.s. \\
\hline \multirow[t]{4}{*}{ Alcohol use } & 0 & $47(44)$ & & $77(71)$ & & $20(19)$ & & $29(27)$ & & $40(39)$ & \\
\hline & 1-4 units/week & $38(40)$ & & $65(68)$ & & $10(11)$ & & $22(23)$ & & $39(43)$ & \\
\hline & 5-10 units/week & $11(31)$ & & $20(57)$ & & $3(9)$ & & $6(17)$ & & $6(18)$ & \\
\hline & 11 or more units/week & $7(24)$ & n.s. & $18(62)$ & n.s. & $0(0)$ & 0,03 & $5(17)$ & n.s. & $8(30)$ & n.s. \\
\hline Anti emetic schedule ${ }^{\mathrm{d}}$ & A & $2(13)$ & & $9(60)$ & & $0(0)$ & & $3(20)$ & & $3(20)$ & \\
\hline \multirow[t]{4}{*}{ Day 1} & $\mathrm{~B}$ & $74(40)$ & & $127(68)$ & & $23(12)$ & & $42(22)$ & & $65(37)$ & \\
\hline & $\mathrm{C}$ & $26(42)$ & & $45(73)$ & & $8(13)$ & & $17(27)$ & & $25(44)$ & \\
\hline & $\mathrm{D}$ & $4(100)$ & & $3(75)$ & & $2(50)$ & & $1(25)$ & & $1(25)$ & \\
\hline & $\mathrm{R}$ & $1(13)$ & n.s. & $4(44)$ & n.s. & $0(0)$ & n.s. & $0(0)$ & n.s. & $1(14)$ & n.s. \\
\hline Anti emetic schedule ${ }^{d}$ & $\mathrm{~B}$ & n.a. & & $43(65)$ & & n.a. & & $19(29)$ & & $21(36)$ & \\
\hline \multirow[t]{4}{*}{ Day 2} & $\mathrm{C}$ & n.a. & & $84(80)$ & & n.a. & & $26(25)$ & & $49(49)$ & \\
\hline & $\mathrm{D}$ & n.a. & & $35(56)$ & & n.a. & & $10(16)$ & & $17(28)$ & \\
\hline & E & n.a. & & $11(61)$ & & n.a. & & $4(22)$ & & $4(22)$ & \\
\hline & $\mathrm{R}$ & n.a. & & $15(60)$ & n.s. & n.a. & & $4(16)$ & n.s. & $4(17)$ & n.s. \\
\hline Anti emetic schedule ${ }^{\mathrm{d}}$ & $\mathrm{B}$ & n.a. & & $27(66)$ & & n.a. & & $11(27)$ & & $14(38)$ & \\
\hline \multirow[t]{4}{*}{ Day 3} & $\mathrm{C}$ & n.a. & & $49(89)$ & & n.a. & & $14(25)$ & & $30(60)$ & \\
\hline & $\mathrm{D}$ & n.a. & & $44(56)$ & & n.a. & & $17(22)$ & & $22(29)$ & \\
\hline & $\mathrm{E}$ & n.a. & & $10(56)$ & & n.a. & & $4(22)$ & & $4(22)$ & \\
\hline & $\mathrm{R}$ & n.a. & & $58(69)$ & 0,03 & n.a. & & $17(20)$ & n.s. & $25(31)$ & 0,006 \\
\hline Total & & $107(39)$ & & $188(68)$ & & $33(12)$ & & $63(23)$ & & $95(34)$ & \\
\hline \multicolumn{12}{|l|}{ Second course $n=253$} \\
\hline \multirow[t]{2}{*}{ Gender } & male & $10(13)$ & & $35(47)$ & & $2(3)$ & & $9(12)$ & & $15(21)$ & \\
\hline & female & $87(49)$ & $<0,005$ & $130(73)$ & $<0,005$ & $22(12)$ & 0,02 & $29(16)$ & n.s. & $75(43)$ & 0,03 \\
\hline \multirow[t]{3}{*}{ Emetogenicity } & HEC & $10(11)$ & & $41(47)$ & & $3(3)$ & & $11(13)$ & & $15(17)$ & \\
\hline & MEC (anthracycline) & $74(55)$ & & $104(78)$ & & $17(13)$ & & $22(16)$ & & $64(48)$ & \\
\hline & MEC (not anthracycline) & $13(41)$ & $<0,005$ & $20(63)$ & 0,03 & $4(13)$ & n.s. & $5(16)$ & n.s. & $11(37)$ & $<0,005$ \\
\hline \multirow[t]{2}{*}{ Age } & $<65$ & $83(44)$ & & $135(71)$ & & $22(12)$ & & $30(16)$ & & $76(41)$ & \\
\hline & $>65$ & $13(21)$ & $<0,005$ & $29(46)$ & $<0,005$ & $2(3)$ & 0,05 & $8(13)$ & n.s. & $13(21)$ & 0,03 \\
\hline Alcohol use & 0 & $35(37)$ & & $57(60)$ & & $12(13)$ & & $12(13)$ & & $33(35)$ & \\
\hline & $1-4$ units/week & $43(46)$ & & $67(71)$ & & $10(11)$ & & $17(18)$ & & $41(45)$ & \\
\hline & 5-10 units/week & $11(33)$ & & $21(64)$ & & $2(6)$ & & $5(15)$ & & $8(25)$ & \\
\hline & 11 or more units/week & $6(23)$ & n.s. & $16(62)$ & n.s. & $0(0)$ & n.s. & $4(15)$ & n.s. & $7(27)$ & n.s. \\
\hline Anti emetic schedule ${ }^{\mathrm{d}}$ & A & $6(32)$ & & $11(58)$ & & $0(0)$ & & $2(11)$ & & $7(37)$ & \\
\hline Day 1 & $\mathrm{~B}$ & $65(39)$ & & $110(67)$ & & $16(10)$ & & $25(15)$ & & $61(37)$ & \\
\hline & $\mathrm{C}$ & $21(39)$ & & $35(65)$ & & $6(11)$ & & $10(19)$ & & $21(41)$ & \\
\hline & $\mathrm{D}$ & $3(50)$ & & $5(83)$ & & $2(33)$ & & $1(17)$ & & $0(0)$ & \\
\hline & $\mathrm{R}$ & $2(20)$ & n.s. & $4(40)$ & n.s. & $0(0)$ & $<0,005$ & $0(0)$ & n.s. & $1(10)$ & n.s. \\
\hline Anti emetic schedule ${ }^{\mathrm{d}}$ & B & n.a. & & $43(75)$ & & n.a. & & $13(23)$ & & $24(42)$ & \\
\hline Day 2 & $\mathrm{C}$ & n.a. & & $65(71)$ & & n.a. & & $15(16)$ & & $38(42)$ & \\
\hline & $\mathrm{D}$ & n.a. & & $31(54)$ & & n.a. & & $5(9)$ & & $16(29)$ & \\
\hline & $\mathrm{E}$ & n.a. & & $10(56)$ & & n.a. & & $2(11)$ & & $7(39)$ & \\
\hline & $\mathrm{R}$ & n.a. & & $16(52)$ & n.s. & n.a. & & $3(10)$ & n.s. & $5(18)$ & n.s. \\
\hline Anti emetic schedule ${ }^{d}$ & $\mathrm{~B}$ & n.a. & & $28(78)$ & & n.a. & & $10(28)$ & & $17(47)$ & \\
\hline
\end{tabular}


Table 3 (continued)

\begin{tabular}{|c|c|c|c|c|c|c|c|c|c|c|c|}
\hline \multirow[t]{4}{*}{ Day 3} & $\mathrm{C}$ & \multicolumn{2}{|l|}{ n.a. } & \multicolumn{2}{|l|}{$30(70)$} & \multicolumn{2}{|l|}{ n.a. } & \multicolumn{2}{|l|}{$6(14)$} & \multicolumn{2}{|l|}{$21(50)$} \\
\hline & $\mathrm{D}$ & \multicolumn{2}{|l|}{ n.a. } & \multicolumn{2}{|l|}{$40(56)$} & \multicolumn{2}{|l|}{ n.a. } & \multicolumn{2}{|l|}{$10(14)$} & \multicolumn{2}{|l|}{$21(30)$} \\
\hline & $\mathrm{E}$ & \multicolumn{2}{|l|}{ n.a. } & \multicolumn{2}{|l|}{$9(56)$} & \multicolumn{2}{|l|}{ n.a. } & \multicolumn{2}{|l|}{$2(13)$} & \multicolumn{2}{|l|}{$6(38)$} \\
\hline & $\mathrm{R}$ & \multicolumn{2}{|l|}{ n.a. } & $58(67)$ & n.s. & \multicolumn{2}{|l|}{ n.a. } & $10(11)$ & n.s. & $25(30)$ & n.s. \\
\hline Total & & \multicolumn{2}{|l|}{$97(38)$} & \multicolumn{2}{|l|}{$165(65)$} & \multicolumn{2}{|l|}{$24(9)$} & \multicolumn{2}{|l|}{$38(15)$} & \multicolumn{2}{|l|}{$90(35)$} \\
\hline \multicolumn{12}{|l|}{ Third course $n=225$} \\
\hline \multirow[t]{2}{*}{ Gender } & male & \multicolumn{2}{|l|}{$10(16)$} & \multicolumn{2}{|l|}{$25(40)$} & $1(2)$ & & $13(21)$ & & $15(25)$ & \\
\hline & female & $83(51)$ & 0,01 & $127(78)$ & $<0,005$ & $20(12)$ & 0,02 & $33(20)$ & n.s. & $69(45)$ & n.s. \\
\hline Emetogenicity & HEC & $7(10)$ & & $35(48)$ & & $1(1)$ & & $15(21)$ & & $14(21)$ & \\
\hline & MEC (anthracycline) & $74(59)$ & & $98(78)$ & & $18(14)$ & & $22(17)$ & & $59(49)$ & \\
\hline & MEC (not anthracycline) & $12(46)$ & $<0,005$ & $19(73)$ & 0,02 & $2(8)$ & 0,01 & $9(35)$ & n.s. & $11(48)$ & 0,02 \\
\hline Age & $<65$ & $82(48)$ & & $126(73)$ & & $19(11)$ & & $34(20)$ & & $71(44)$ & \\
\hline & $>65$ & $10(20)$ & $<0,005$ & $25(48)$ & n.s. & $1(2)$ & 0,05 & $12(23)$ & n.s. & $12(26)$ & 0,05 \\
\hline Alcohol use & 0 & $35(42)$ & & $55(65)$ & & $11(13)$ & & $18(21)$ & & $36(47)$ & \\
\hline & 1-4 units/week & $44(51)$ & & $64(74)$ & & $8(9)$ & & $18(21)$ & & $27(45)$ & \\
\hline & 5-10 units/week & $7(25)$ & & $18(64)$ & & $2(7)$ & & $8(29)$ & & $7(25)$ & \\
\hline & 11 or more units/week & $5(25)$ & 0,02 & $10(50)$ & n.s. & $0(0)$ & n.s. & $2(10)$ & n.s. & $2(11)$ & n.s. \\
\hline Anti emetic schedule ${ }^{\mathrm{d}}$ & A & $6(40)$ & & $10(67)$ & & $0(0)$ & & $2(13)$ & & $4(29)$ & \\
\hline Day 1 & $\mathrm{~B}$ & $65(43)$ & & $99(65)$ & & $16(11)$ & & 27 (18) & & $58(40)$ & \\
\hline & $\mathrm{C}$ & $17(36)$ & & $37(79)$ & & $5(11)$ & & $15(32)$ & & $20(47)$ & \\
\hline & $\mathrm{D}$ & $2(50)$ & & $3(75)$ & & $0(0)$ & & $1(25)$ & & $0(0)$ & \\
\hline & $\mathrm{R}$ & $3(50)$ & n.s. & $3(43)$ & n.s. & $0(0)$ & n.s. & $1(14)$ & n.s. & $2(40)$ & n.s. \\
\hline Anti emetic schedule ${ }^{\mathrm{d}}$ & $\mathrm{B}$ & n.a. & & $40(69)$ & & n.a. & & $13(22)$ & & $29(51)$ & \\
\hline Day 2 & $\mathrm{C}$ & n.a. & & $53(80)$ & & n.a. & & $17(26)$ & & $31(50)$ & \\
\hline & $\mathrm{D}$ & n.a. & & $34(61)$ & & n.a. & & $10(18)$ & & $17(31)$ & \\
\hline & $\mathrm{E}$ & n.a. & & $10(67)$ & & n.a. & & $1(7)$ & & $3(23)$ & \\
\hline & $\mathrm{R}$ & n.a. & & $15(50)$ & n.s. & n.a. & & $5(17)$ & n.s. & $4(16)$ & 0,02 \\
\hline Anti emetic schedule ${ }^{\mathrm{d}}$ & $\mathrm{B}$ & n.a. & & $23(68)$ & & n.a. & & $9(26)$ & & $18(53)$ & \\
\hline Day 3 & $\mathrm{C}$ & n.a. & & $32(86)$ & & n.a. & & $11(30)$ & & $20(59)$ & \\
\hline & $\mathrm{D}$ & n.a. & & $40(59)$ & & n.a. & & $12(18)$ & & $20(30)$ & \\
\hline & $\mathrm{E}$ & n.a. & & $8(73)$ & & n.a. & & $0(0)$ & & $2(20)$ & \\
\hline & $\mathrm{R}$ & n.a. & & $29(65)$ & n.s. & n.a. & & 14 (19) & n.s. & $24(36)$ & n.s. \\
\hline Total & & $93(41)$ & & $152(68)$ & & $21(9)$ & & $46(20)$ & & 84 (37) & \\
\hline
\end{tabular}

${ }^{\mathrm{a}}$ based on logisitic regression analysis

${ }^{\mathrm{b}}$ based on chi2 analysis

${ }^{c}$ the numbers and percentages present patients with an impact on daily life, a FLIE score of $<108$ indicaties an impact on daily life

${ }^{d}$ schedule A is the cmbination of a 5HT3 antagonist, aprepitant and a corticosteroid

schedule B is the combination of a 5HT3 antagonist and a corticosteroid

schedule $\mathrm{C}$ is a $5 \mathrm{HT} 3$ antagonist

schedule D is a corticosteroid

schedule $\mathrm{E}$ is aprepitant and a corticosteroid (day 2 and 3 )

schedule $\mathrm{R}$ is other anti emetics (for exemple metoclopramide or benzodiazepine)

n.s. not significant

n.a. not applicable 
associations were observed between alcohol use and CINV.

\section{Emetogenicity of chemotherapy and CINV (Table 3)}

At all treatment cycles, significantly more patients receiving moderately emetogenic chemotherapy, containing anthracycline, reported acute nausea than patients receiving highly emetogenic chemotherapy (first cycle $59 \%$ vs $14 \%$, $p<0.005$; second cycle $55 \%$ vs $11 \%, p<0.005$; third cycle $59 \%$ vs $10 \%, p<0.005)$. In addition, significantly more patients with moderately emetogenic chemotherapy, containing anthracycline, experienced acute vomiting during the first and third treatment cycle ( 19 vs $3 \%, p<0.005$ and 14 vs $1 \%, p=0.01)$. At the second and third cycle, significantly more patients receiving moderately emetogenic chemotherapy, containing anthracycline, suffered from delayed nausea ( $78 \%$ vs $47 \%, p=0.03$ and 78 vs $48 \%, p=$ 0.02 ). Also, significantly more patients receiving moderately emetogenic chemotherapy, containing anthracycline, reported that CINV had a negative impact on daily life at all treatment cycles (first cycle $43 \%$ vs $25 \%, p=0.02$; second cycle $48 \%$ vs $17 \%, p<0.005$; third cycle 49 vs $21 \%, p=0.02$ ).

\section{Antiemetic use and CINV (Table 3)}

As described in the methods section, the different combinations of antiemetics were classified in six different groups paralleling the combinations of antiemetics described in the international guidelines summarized in Table 1 [1].

HEC In our study, $15 \%$ of patients receiving HEC were treated with antiemetics containing aprepitant, a 5HT3 antagonist and dexamethason; $55 \%$ of the HEC patients were treated with a combination of a 5HT3 antagonist and dexamethason, and $25 \%$ with a $5 \mathrm{HT} 3$ antagonist as a single agent. On day 2, 30\% of the HEC patients were treated with a combination of a 5HT3 antagonist and a corticosteroid, $24 \%$ with a 5HT3 antagonist alone, and $16 \%$ with a corticosteroid alone. On day 3 , these combinations of antiemetics were used in $24 \%, 16 \%$, and $22 \%$ of cases, respectively. Patients treated with a combination of antiemetics containing aprepitant on day 1 , were also treated with aprepitant on days 2 and 3. The remaining patients were treated with other types of antiemetics, e.g., metoclopramide, benzodiazepines.

$M E C$ No MEC-A patients were treated with aprepitant; $80 \%$ of these patients were treated with a combination of a 5HT3 antagonist and dexamethason for acute CINV.

On day 2, 20\% of the MEC-A patients were treated with the combination of a 5HT3 antagonist and dexamethason, $46 \%$ were treated with a $5 \mathrm{HT} 3$ antagonist as single agent, and $29 \%$ with dexamethason as single agent. On the third day after chemotherapy, the percentages on these treatment combinations were $10 \%, 21 \%$ and $34 \%$, respectively. The remaining patients were treated with other types of antiemetics, e.g., metoclopramide, benzodiazepines.

In general, very few statistically significant associations were observed between type of antiemetic used and CINV. Eighty-nine percent of patients using a 5HT3 antagonist at the third day after the first cycle of chemotherapy suffered from delayed nausea, compared to $56 \%$ of patients using a combination of aprepitant and a corticosteroid or a corticosteroid as a single agent $(p=0.03)$. This was also reflected in the percentage of patients in these two subgroups reporting CINV-related effects on their daily lives $(60 \%$ vs $22 \%, p=0.006)$. At the second treatment cycle, no patient using a combination of antiemetics (aprepitant, 5HT3 antagonist, and a corticosteroid) suffered from acute vomiting, compared to $33 \%$ of patients using a corticosteroid $(p<0.005)$.

\section{Adding antiemetics between treatment cycles (Table 4)}

To evaluate changes in (i.e., the addition of) antiemetics between treatment cycles and the factors affecting those changes, we compared the (combination of) antiemetics used on day 1 of the first cycle with those used on day 1 of cycles 2 and 3. The same comparison was done for the antiemetics used on days 2 and 3 of the first cycle with those used on days 2 and 3 of cycles 2 and 3 .

Table 4 shows the results of the logistic regression analysis based on patients for whom additional antiemetics were added over the course of their treatment. On the first day of the treatment cycle (the day the chemotherapy was administered), patients suffering from acute vomiting were significantly more likely to receive extra antiemetics than patients not suffering from these symptoms (acute vomiting 34 vs $14 \%, p=0.009$ ). However, the experience of CINV on the second and third day after the administration of chemotherapy did not result in the use of additional antiemetics.

On day 1, 42 patients received additional antiemetics; $64 \%$ of these patients received extra corticosteroids and $17 \%$ aprepitant. On day 2, 43 patients received additional antiemetics, of whom $56 \%$ were treated with extra corticosteroids and $21 \%$ with $5 \mathrm{HT} 3$ antagonist. On day 3, 54 patients received additional antiemetics, of whom $35 \%$ were treated with a $5 \mathrm{HT} 3$ antagonist, and $35 \%$ with a corticosteroid. The remaining patients were treated with other extra types of antiemetics, e.g., metoclopramide, benzodiazepines. Logistic regression analysis did not show improved symptom control due to the addition of these antiemetics. 
Table 4 Association between CINV symptoms and use of additional antiemetic medications during subsequent chemotherapy cycles $(n=225)$

\begin{tabular}{|c|c|c|c|c|c|c|c|c|c|c|}
\hline & & $\begin{array}{l}\text { Day } 1 \\
n(\%)\end{array}$ & OR & $p$ value & $\begin{array}{l}\text { Day } 2 \\
n(\%)\end{array}$ & OR & $p$ value & $\begin{array}{l}\text { Day } 3 \\
n(\%)\end{array}$ & OR & $p$ value \\
\hline \multirow[t]{2}{*}{ Age } & $<65$ years & $31(16)$ & & & $33(17)$ & & & $40(21)$ & & \\
\hline & $>65$ years & $11(17)$ & 1 & 0.97 & $8(13)$ & 1 & 0.27 & $13(21)$ & 1 & 0.81 \\
\hline \multirow[t]{2}{*}{ Sex } & Male & $10(13)$ & & & $9(12)$ & & & $11(15)$ & & \\
\hline & Female & $32(18)$ & 0.7 & 0.41 & $33(18)$ & 0.7 & 0.54 & $42(23)$ & 0.6 & 0.28 \\
\hline \multirow[t]{3}{*}{ Emetogenicity } & HEC & $16(18)$ & & & $12(14)$ & & & $13(15)$ & & \\
\hline & $\begin{array}{l}\text { MEC with } \\
\text { anthracycline }\end{array}$ & 24 (18) & & & $28(21)$ & & & $36(27)$ & & \\
\hline & $\begin{array}{l}\text { MEC without } \\
\text { antracycline }\end{array}$ & $2(6)$ & 0.57 & 0.11 & $2(6)$ & 0.7 & 0.3 & $4(13)$ & 1 & 0.95 \\
\hline \multirow[t]{4}{*}{ Alcohol use } & 0 units/week & $15(16)$ & 0.4 & & $15(16)$ & 0.7 & & 18 (19) & 0.8 & \\
\hline & 1-4 units/week & $13(14)$ & 0.5 & & $17(18)$ & 0.8 & & $24(26)$ & 1.2 & \\
\hline & 5-10 units/week & $6(18)$ & 0.6 & & $3(9)$ & 0.4 & & $5(15)$ & 0.6 & \\
\hline & $\begin{array}{l}11 \text { or more } \\
\text { units/week }\end{array}$ & $6(23)$ & & 0.52 & $5(19)$ & & 0.67 & $5(19)$ & & 0.53 \\
\hline \multirow{2}{*}{$\begin{array}{l}\text { Acute vomiting } \\
1 \text { st course }\end{array}$} & Yes & $10(34)$ & & & $8(28)$ & & & $8(28)$ & & \\
\hline & No & $32(14)$ & 5.7 & 0.009 & $34(15)$ & 0.5 & 0.29 & $45(20)$ & 0.4 & 0.17 \\
\hline \multirow{2}{*}{$\begin{array}{l}\text { Acute vomiting } \\
\text { 2nd course }\end{array}$} & Yes & $4(17)$ & & & $5(21)$ & & & $5(21)$ & & \\
\hline & No & $38(17)$ & 0.4 & 0.56 & 37 (16) & 1.5 & 0.56 & $48(21)$ & 2.1 & 0.29 \\
\hline \multirow{2}{*}{$\begin{array}{l}\text { Delayed vomiting } \\
1 \text { st course }\end{array}$} & Yes & $28(14)$ & & & $31(16)$ & & & $43(22)$ & & \\
\hline & No & $14(24)$ & 1.5 & 0.4 & 11 (19) & 0.8 & 0.57 & $10(17)$ & 0.43 & 0.06 \\
\hline \multirow{2}{*}{$\begin{array}{l}\text { Delayed vomiting } \\
\text { 2nd course }\end{array}$} & Yes & $35(16)$ & & & $32(15)$ & & & $41(19)$ & & \\
\hline & No & $7(18)$ & 1 & 0.96 & $10(26)$ & 2.2 & 0.12 & $12(32)$ & 2.3 & 0.07 \\
\hline \multirow{2}{*}{$\begin{array}{l}\text { Acute nausea } \\
1 \text { st course }\end{array}$} & Yes & $23(15)$ & & & $22(14)$ & & & $29(18)$ & & \\
\hline & No & $19(20)$ & 0.8 & 0.73 & $20(21)$ & 0.6 & 0.34 & $24(25)$ & 1.1 & 0.88 \\
\hline \multirow{2}{*}{$\begin{array}{l}\text { Acute nausea } \\
\text { 2nd course }\end{array}$} & Yes & $26(17)$ & & & $19(12)$ & & & $31(20)$ & & \\
\hline & No & $16(16)$ & 2.3 & 0.11 & $23(23)$ & 2.4 & 0.09 & $22(23)$ & 0.7 & 0.39 \\
\hline \multirow{2}{*}{$\begin{array}{l}\text { Delayed nausea } \\
1 \text { st course }\end{array}$} & Yes & $7(8)$ & & & $10(12)$ & & & $12(14)$ & & \\
\hline & No & $35(20)$ & 2.6 & 0.07 & $32(19)$ & 1.3 & 0.59 & $41(24)$ & 1.7 & 0.24 \\
\hline \multirow{2}{*}{$\begin{array}{l}\text { Delayed nausea } \\
\text { 2nd course }\end{array}$} & Yes & $12(13)$ & & & $11(12)$ & & & $14(16)$ & & \\
\hline & No & $30(18)$ & 1.1 & 0.83 & 31 (19) & 0.8 & 0.63 & $39(24)$ & 1.2 & 0.76 \\
\hline
\end{tabular}

\section{Discussion}

Chemotherapy-induced nausea and vomiting have a clear impact on the daily lives of cancer patients. Antiemetic therapy is aimed at preventing, ameliorating or minimizing CINV. Most studies of the effects of CINV have been characterized by narrow patient selection criteria and have investigated well-defined, protocol-based chemotherapy, and antiemetic regimens.

The results of this study of CINV and antiemetic use in routine clinical practice indicate that CINV and particularly delayed symptoms, impact significantly on patients' daily life, despite the use of different combinations of antiemetics. Our findings are consistent with those of other studies, in which the prevalence of delayed nausea and vomiting is higher than acute nausea and vomiting [5].

However, contrary to expectations, we found that patients receiving treatment with moderately emeto- genic chemotherapy containing anthracyclines (MECA) suffered more from CINV than patients receiving HEC.

A previous study in daily clinical practice by BloechlDaum reported no significant differences in the rate of acute and delayed nausea between patients treated with HEC and MEC, while significantly more patients receiving HEC than MEC reported that CINV had a negative impact on daily life $(52.8 \%$ and $35.5 \%, p=$ 0.027) [5]. However, Mollassiotis et al. reported that patients receiving MEC experienced higher levels of CINV compared to patients receiving HEC in a large, single center study in the UK [21]. These two studies were conducted before antiemetic guidelines incorporated the use of NK-1 receptor antagonist containing regimens for patients receiving HEC. After completion of our study, antiemetic guidelines for MEC-A were also updated. Currently, a three-drug combination regimen, including 
an NK-1 receptor antagonist, is also recommended for MEC-A regimens [1]. Our finding that patients who received $\mathrm{MEC}$ treatment faired particularly poorly in terms of CINV symptoms may be explained, at least in part, by the fact that these patients did not receive newer and more effective antiemetic treatment including aprepitant. Additional studies are needed to determine the effectiveness in daily clinical practice of newer antiemetic treatments among patients receiving MEC-A.

Various other factors, in addition to the emetogenicity of the chemotherapy, have been identified that influence both the prevalence and the severity of CINV. These include age, gender, alcohol use, history of motion sickness, and history of pregnancy-related vomiting. In our study, the first three of these risk factors were found to be associated significantly with CINV, but not the latter two. It is unclear why this is the case. It may be that once female gender, which was a strong predictor variable, entered the logistic regression model, motion sickness and pregnancy-related vomiting did not have any additional predictive value.

Interestingly, in a recent analysis of data from two phase III trials of aprepitant in patients receiving cisplatin-based chemotherapy, female gender was not found to be a significant risk factor for CINV [22]. It would be of interest to determine if this also holds true for patients receiving MEC-A, because the majority of these patients are women being treated for breast cancer with anthracyclinecontaining regimens.

Given the finding that patients lost to follow-up were younger and significantly more likely to receive HEC, patients' level of CINV after multiple treatment cycles might be underestimated in our study because of the increased risk of CINV associated with these risk factors.

The current study is, to the best of our knowledge, the first to investigate change in antiemetic use, and specifically, the use of additional antiemetics, during consecutive cycles of chemotherapy. Previous studies on CINV over multiple treatment cycles have been carried out in welldefined patient populations using predefined antiemetic regimens that were held constant throughout the chemotherapy treatment [23]. In our study, we found that patients experiencing acute vomiting were significantly more likely to have antiemetics added in subsequent chemotherapy cycles than those not experiencing acute vomiting. However, no significant association was observed between other symptoms of CINV and the use of additional antiemetics during subsequent treatment cycles. Additionally, our results indicate that delayed CINV had no effect on subsequent antiemetic treatment. This may be due, in part, to the fact that physicians and nurses often underestimate the incidence of delayed nausea and vomiting [13]. The introduction of standardized, clinic- and home-based symptom monitoring (the latter via interactive voice recognition systems or internet-based data capture) could provide health care providers with real time information on delayed CINV, which, in turn, could have a salutary effect on symptom management.

Conflict of interest The authors have no conflict of interest to declare.

Open Access This article is distributed under the terms of the Creative Commons Attribution Noncommercial License which permits any noncommercial use, distribution, and reproduction in any medium, provided the original author(s) and source are credited.

\section{Appendix}

Contributing hospitals, hospital pharmacists, and oncology nurses: Diaconessenhuis Leiden: G. Wassink, D. van Hooff, E. Kulk; Gemini Ziekenhuis: G. Nap, R. Timmerman, E. Beer; Medisch Centrum Alkmaar: P. Kloeg, S. de Bie, M. Jong; Onze Lieve Vrouwe Gasthuis: C. van der Linden, M. Attema-de jonge, J.M. Jenje, P.W. van der Velde, N.W.M. Gerrits, E.B. Gooszen, B. de Valk; Rijnland ziekenhuis:J.M.R. Hollander, I. Borst, E. van Belsen; Sint Lucas Andreas ziekenhuis: J. van den Heuvel, P. den Hartogh, J. van de Klooster, G. Kuper, K. Keijzer, E. vd Boogaard; Waterland ziekenhuis; R. de Jong, C. Boers; WestFries Gasthuis: B. van der Kleij, M. Plas, B. Vonk, M. Dudink; Zaans Medisch Centrum: M.G. Schuitenmaker, C. Bakker, M. Termeulen, M. de Waard

\section{References}

1. Antiemesis. NCCN practice guidelines in oncology-v.1.2007. www.ncen.org

2. Hesketh PJ, Gandara DR (1991) Serotonin antagonists: a new class of antiemetic agents. J Natl Cancer Inst 83:613-620

3. Hesketh PJ, Grunberg SM, Gralla RJ et al (2003) The oral neurokinin-1 antagonist aprepitant for the prevention of chemotherapy-induced nausea and vomiting: a multinational, randomized, double-blind, placebo-controlled trial in patients receiving high-dose cisplatin-the Aprepitant Protocol 052 Study Group. J Clin Oncol 21:4112-4119

4. De Wit R, Herrstedt J, Rapoport B et al (2004) The oral NK(1) antagonist aprepitant, given with standard antiemetics provides protection against nausea and vomiting over multiple cycles of cisplatin-based chemotherapy. Eur J Cancer 40:403-410

5. Bloechl-Daum B, Deuson RR, Mavros P, Hansen M, Herrstedt J (2006) Delayed nausea and vomiting continue to reduce patients' quality of life after highly and moderately emetogenic chemotherapy despite antiemetic treatment. J Clin Oncol $24: 4472-4478$ 
6. D'Acquisto R, Tyson LB, Gralla RJ (1986) The influence of a chronic high alcohol intake on chemotherapy-induced nausea and vomiting. Proc Am Soc Clin Oncol 5:257

7. Hesketh PJ, Plagge P, Bryson JC (1992) Single-dose ondansetron for prevention of acute cisplatin-induced emesis: analysis of efficacy and prognostic factors. In: Bianchi AL, Grelot L, Miller Ad (eds) Mechanisms and control of emesis. Libbey, London, pp 25-26

8. Pollera CF, Giannarelli D (1989) Prognostic factors influencing cisplatin-induced emesis. Definition and validation of a predictive logistic model. Cancer 64:1117-1122

9. Tonato M, Roila F, Del Favero A (1991) Methodology of antiemetic trials: a review. Ann Oncol 2:107-114

10. Booth CM, Clemons M, Dranitsaris G, Joy A, Young S, Callaghan W, Trudeau M, Petrella T (2007) Chemotherapyinduced nausea and vomiting in breast cancer patients: a prospective observational study. J Support Oncol 5:374-380

11. Osoba D, Zee B, Pater J, Warr D, Latreille J, Kaizer L (1997) Determinants of postchemotherapy nausea and vomiting in patients with cancer. Quality of Life and Symptom Control Committees of the National Cancer Institute of Canada Clinical Trials Group. J Clin Oncol 15:116-123

12. Schwartzberg LS (2007) Chemotherapy-induced nausea and vomiting: clinician and patient perspectives. J Support Oncol 5:5-12

13. Grunberg SM, Deuson RR, Mavros P, Geling O, Hansen M, Cruciani G, Daniele B, De Pouvourville G, Rubenstein EB, Daugaard G (2004) Incidence of chemotherapy-induced nausea and emesis after modern antiemetics. Perception versus reality. Cancer 100:2261-2268

14. Gralla RJ, Osoba D, Kris MG et al (1999) Recommendations for use of antiemetics: evidence-based clinical practice guidelines. J Clin Oncol 17:2971-2994
15. Kris MG, Hesketh PJ, Somerfield MR et al (2006) American Society of Clinical Oncology guideline for antiemetics in oncology: update 2006. J Clin Oncol 24:2932-2947

16. Lindley CM, Hirsch JD, O'Neill CV et al (1992) Quality of life consequences of chemotherapy-induced emesis. Qual Life Res $1: 331-340$

17. Martin AR, Pearson JD, Cai B et al (2003) Assessing the impact of chemotherapy-induced nausea and vomiting on patients' daily lives: a modified version of the Functional Living Index-Emesis (FLIE) with 5-day recall. Support Care Canc 11:522-527

18. Ballatori E, Roila F (2003) Impact of nausea and vomiting on quality of life in cancer patients during chemotherapy. Health Qual Life Outcomes 1:46

19. Hesketh PJ (1999) Defining the emotogenicity of cancer chemotherapy regimens: relevance to clinical practice. Oncologist 4:191-196

20. McNemar's test for correlated proportions. Available from URL: http://faculty.vassar.edu/lowry/VassarStats.html. Accessed 28 December 2009

21. Molassiotis A, Saunders MP, Valle J, Wilson G, Lorigan P, Wardley A, Levine E, Cowan R, Loncaster J, Rittenberg C (2008) A prospective observational study of chemotherapy-related nausea and vomiting in routine practice in a UK cancer centre. Support Care Canc 16:201-208

22. Hesketh PJ, Aapro M, Street J, Carides A (2009) Evaluation of risk factors predictive of nausea and vomiting with current standard-of-care antiemetic treatment: analysis of two phase III trials of aprepitant in patients receiving cisplatin-based chemotherapy. Support Care Cancer 18:1171-1177

23. Ellebaek E, Herrstedt J (2008) Optimizing antiemetic therapy in multiple-day and multiple cycles of chemotherapy. Curr Opin Support Palliat Care 2:28-34 